\title{
The Double Wall Boron Nitride Nanotube: Nano-Cylindrical Capacitor
}

\author{
MARZIEH SADAT MADANI, MAJID MONAJJEMI* and HOSSEIN AGHAEI \\ Department of Chemistry, Science and Research Branch, Islamic Azad University, Tehran, Iran. \\ *Corresponding author E-mail :m_monajjemi@srbiau.ac.ir
}

http://dx.doi.org/10.13005/ojc/330320

(Received: February 17, 2017; Accepted: March 13, 2017)

\begin{abstract}
Experimental results have shown that a small single wall carbon Nanotubes (SWCNTs) can be usually found inside "multi-walled" (MWCNTs). In this work it has been reported the stabilities and electronic structure of the single wall boron nitride Nano-tube (SWBNNTs) inside SWBNNTs. It has been shown that the gap energies and that energy of BNNTs are strongly dependent on their diameters or chirality. When this kind of BNNTs are inserted in the larger one of SWBNNTs, the gap energies of the double walled (DWBNNTs) would even be much decreased due to the "coupled effect" of "wall buckling" difference and inter-wall $\pi-\pi^{\star}$ "hybridization". DWBNNTs are used for a theoretical study of a cylindrical molecular capacitor, including an inner cylinder with a positive charge distribution and an outer cylinder with a negative charge distribution. Due to their semiconductor characteristic and dielectric functionalities of SWBNNTs, DWBNNTs can be used as a cylindrical capacitor for the electronic devices.
\end{abstract}

Keyword: single-wall BN-nanotubes, nano-cylindrical capacitors

\section{INTRODUCTION}

NSCCE or "Nanometer-scale capacitive charging effect" currently is familiar, e.g., the "coulomb blockade phenomenon" in the quantum $\operatorname{dots}^{1,2}$. However, they are arduous for resolving the detailed radial charges repartition within the nanostructures. Using SWBNNTs@SWBNNTs double wall tubes; it has been discussed the "radial" charge distributions of multi layered molecular capacitors.

Designing and control of Nano-tubes diameters are requirement to develop Nano-tube growth method. Nano-tubes with the diameters of less than one nanometer provide the ideal Nanospaces in X-dimension ${ }^{3}$. A few years ago, it has been suggested which the sizes of the catalysts used in the metal catalyze CVD (chemical vapor deposition) can explain the diameter of grown carbon nanotube ${ }^{4}$. Thisopinion has been confirmed by the seeing that a catalytic particle in the end of chemical vapor deposition grown nanotube has size proportional with the Nano-tubes diameters ${ }^{5}$.

Chin Li Cheung, illustrate clearly the concepts of different sizes nano cluster catalysts which they can be used for controlling the structures 
and diameters of "CVD" grown nanotubes" 6 . Smalldiameter carbon nanotubes indicate to many exotic properties such as an-isotropic optical absorption spectra ${ }^{7}$ and super conductivities emanate from a "Pearle" distortion"8,9. This finding has stimulated much fondness in studies of a small nanotube in both theoretically and experimentally ${ }^{10-14}$.BN-Nanotubes(BNNTs), which firstly synthesized and predicted through Chopra work and Rubiorespectively ${ }^{15,16}$, has a unique structural syllogism to carbon Nano-tubes but, contrary to the $\mathrm{C}-\mathrm{N}-\mathrm{T}$ being semiconductor or metallic depending on their chirality ${ }^{16}$. BNNT is usually can be used as an insulator regardless of its diameter and helicity or the number of walls ${ }^{15-17}$.

Experimental result has shown that a small SWNT is usually found inside a multi-walled $\mathrm{CNT}^{11-13}$. Therefore, there are strong incentive for studding in details the stabilities and interaction of narrow BNNT inside a bigger one in viewpoint of diameter, which makes easier for understanding the experimental result. Furthermore, the studies of double-walled boron nitride Nano-tubes (DBNNTs) have displayed interesting variations in its electronic properties comparing with those of free-standing part of BNNTs ${ }^{18}$. So it is also significant for seeing the interaction energies associated and inter wall coupling behavior with the narrow BNNTs ${ }^{17,18}$.

B-Nnanotube possessesgrate band gap around5 to $6 \mathrm{eV}$ regardless of diameters, chirality, electronic properties and the number of walls15, 19.Furthermore, they are stable in view-point of mechanical and chemical structures ${ }^{20}$. Therefore, narrow single-wall BNNT can widely be applies as an ideal Nano-tube for the Nanosciences for producing suitable material such as; capacitor, atomic wire, and semiconductor ${ }^{21}$, 22.In our study the SWBNNs are special material as an insulator for producing Nano-cylindrical capacitors ${ }^{22}$.

Ryo Nakanishi reported an important synthesis method of a narrow SWBNNT having uniform distribution of diameter around $0.7 \pm 0.1$ $\mathrm{nm}^{1}$. Their strategies to synthesize thin BNNTs are for combining the Nano-template reaction using Single wall carbon Nano-tubes which has developed in the past ten years ${ }^{23}$. In their systems, precursor molecules including Ammonia Borane Complexes or $\mathrm{ABC}$, including boron and nitrogen were en-capsulated first in the single wall carbon tubes followed by suitable thermal decomposition-fusion ${ }^{22}$ reaction inside the SWCNTs ${ }^{22,23}$.

It has been used arc-grownSWCNTs ${ }^{24}$ with distribution (diameter)around1.4 $\mathrm{nm}^{24}$ as a model for synthesizing narrow SWBNNT ${ }^{22,24}$. Those distributions of SWCNT-models are essential for realizing the diameter selective synthesizing of BNNTs.

In this investigation, the systems have been simulated based on the various distribution diameters of SWBNNTs @SWBNNTs corresponding the experimental results of Ryo Nakanishiresults ${ }^{1}$.So we have started for answering to some questions for the mechanism of the radial charge distributions on the inner and outer electrodes, band gapes, potential difference ${ }^{23}$ between two layers of the Nano cylindrical ${ }^{24}$ capacitor and the capacitance of our system when the inner tubes are semiconducting ${ }^{25}$, and the others are metallic ${ }^{26}$.

Gugang Chen in the studies of doped double-walled carbon nanotubes ${ }^{26}$ exhibited the Resonant-Raman-Scattering (RRS) from the phonons $^{26}$ on each carbon shell determines the radial charges distributions ${ }^{26}$. The self-consistent including tight-binding model (SCTB) conûrms ${ }^{25}$ the observed molecular faraday-cage effects, so most of the charges reside on the outer-walls, even when these walls were originally ${ }^{26}$ semiconducting and the inner-walls were metallic ${ }^{24-26}$.

Those systems have been modeled as three-layer cylindrical capacitor within bromine anion forming the shell (around the outer nanotubes ${ }^{26}$ ). The total energies contain3terms including the innertube, outer tube and band structures of the electrostatic energies $\mathrm{E}^{\mathrm{es}}$ for the three-layer charge distributions: $\Sigma_{\mathrm{i}, \mathrm{k}} \mathrm{E}_{\mathrm{i}}^{\text {inner }}(\mathrm{k})+\mathrm{E}_{\mathrm{i}}^{\text {outer }}(\mathrm{k})+\mathrm{E}_{\mathrm{es}}$

The signs" " and " $k$ " label the wave vector and occupied band for the outer or inner tube $e^{26}$.

They assumed that the surpluses charges on both shells are distributed in the innitely thin-walls at the nuclear radius of those shell ${ }^{26,27}$. The resulting electrostatic energies of the triple-walled capacitors are: $E^{e s}=1 / 2 e^{2} L / 2 \pi \varepsilon_{0} n^{2}{ }_{\text {inner }} I_{n}\left(R_{\text {outer }} R_{\text {inner }}\right)+1 / 2 e^{2} L /$ 
$2 \pi \varepsilon_{0} n^{2} I_{n}\left(R_{B r} R_{\text {inner }}\right)$ where $\left\{\varepsilon_{0}\right\}$ is the permittivity of free space $^{27}, L$ is the unit-cell length of the outer tubes and $(n)$ is the linear densities of surpluses holes for the innertubes.

There are two physical properties acts in concert ${ }^{27}$ for isolating most of the holes into the outer nanotubes.(1): the gap band of the thinner diameter tubes towards the larger one, so they empty last $^{28}$. (2) The cylindrical-geometry rather raises the electrostatics potentials in the inner tubes ${ }^{27}$. Lonely the charges on the inner tubes in the chirality of Zigzag $(n, 0)$ of BNNTs are anticipated to have direct band gaps ${ }^{27}$. On the other side the armchair $(n, n)$ of BNNTs will have indirect band gaps ${ }^{27}$. Because of its large band gap around $5 \mathrm{eV}$, experiments ${ }^{28}$ using BNNT as the conduction-channel ${ }^{29}$ for field effect transistors ${ }^{29}$ or FETs showed that BNNT allowed transport through only the valence band ${ }^{28}$. The other important features about the band gaps of BNNTs are that they are tunable by doping with carbons ${ }^{29}$, radial deformation ${ }^{30}$, or by applying the transverses electric fields through the BNNTs so-called giantstark-effect ${ }^{31-33}$.

Theoretical band structure calculation suggested that "SWBNNT" can either be n-type or p-type semiconductor by controlling the composition of carbon into "SWBNNTs". Carbon impurities on a boron site result in electron carriers while on a nitrogen site result in holecarriers ${ }^{34}$.

In this study it has been exhibited that the piezo electricity ${ }^{34}$ for SWBNNTs causes for increasing the capacities of SWBNNTs@SWBNNTs capacitor comparing to SWCNTs@SWCNTs. This phenomenon ${ }^{35}$ originated from the deformation effects due to the tumbling of the planar hexagonal boron nitride network to produce tubular structures ${ }^{35}$. It has been exhibited by Nakhmans on that BNNT could be excellent piezoelectric systems ${ }^{36}$. As instant, piezoelectric constants for variant zigzags of SWBNNT were found for increasing along with the decreasing of the radius in several BNNTs ${ }^{36}$. Experimentally ${ }^{37}$, Bai has exhabited that under in situ elastic bending deformation ${ }^{36}$ or EBD at room temperature high-resolution-transmission-electronmicroscope ${ }^{36,37}$, a normally electrically insulating ${ }^{37}$ MWBNNT may transform to a semiconductor ${ }^{37}$.
K. Uchida et al., ${ }^{38-39}$ in a discussion of quantum effect in the cylindrical carbon nanotubes capacitor exhibited that the distributions of the accumulated charges in the inner tubes are quantum mechanically ${ }^{37}$ spilled outward, while that in the outer tubes are penetrating inwards ${ }^{38}$. They have shown the reflecting those charges spills, the electrostatic capacitance of the systems are larger than what would be expected from the classical theories ${ }^{38}$.

Finally, they have shown that the capacitance exhibit two principal quantum effects, (1): the capacitance shows a large bias dependence ${ }^{38}$, reflecting the densities of states of the carbon Nano tube electrodes. (2): the capacitances are enhanced according to a quantum mechanical spill of the stored electrons density from the tubes walls of the $\mathrm{CNTs}^{36-38}$.

Based on our previous works ${ }^{40-61}$, we simulated our model in viewpoint of different band gap energies via considering the single wall boron nitrides as both inner and outer tubes with variant diameters and chirality in the ranges of $(6.0<\mathrm{d}<8.0 \mathrm{~A})$ and $(11.0<\mathrm{d}<16.0 \mathrm{~A})$ for inner and outer tubes respectively.

\section{Theoretical background}

Carbon nanotube is thin seam less graphitic cylinders $^{39}$, which exhibit an unusual combination of the Nano-meter size diameter and Milli-meter size length ${ }^{39}$. These topologies, are included with the absence of defects ${ }^{38}$ on the macroscopic scales $^{62}$, yields to uncommon electronic properties of individual62,63 SWCNTsthat depends on their diameters and chirality ${ }^{63}$, can be either ${ }^{63}$ insulating, metallic or semiconducting ${ }^{64,65}$.

Consider a cylindrical capacitor of length " $\mathrm{d}_{1}$ ", inner radius " $\mathrm{R}_{\mathrm{inn}}$ ", outer radius " $\mathrm{R}_{\text {out }}$ ", and with charges $Q=d_{1}, q_{\lambda}$ which $q_{\lambda}$ is the charges per unit length (magnitude) on each cylinders (Scheme1). Assume " $\mathrm{d}_{1}$ " $>>$ " $\mathrm{R}_{\mathrm{inn}}$ " or " $\mathrm{R}_{\text {out }}$ " and Neglect fringing and electric elds between cylinders: use Gauss' law $E\left(2 \pi r d_{1}\right)=d_{1} \cdot q_{\lambda} / \varepsilon_{o}=E(r)=q_{\lambda}, 2 \pi \varepsilon_{0} r$ (1) and electric potentials between cylinders: use $\mathrm{V}_{\text {out }}=0$ $\mathrm{V}(\mathrm{r})=-\int_{R_{\text {out }}}^{r} E(r) d r=-\frac{q_{\lambda}}{2 \pi \varepsilon_{0}} \int_{o}^{r} \frac{d r}{r}=-\frac{q_{\lambda}}{2 \pi \varepsilon_{0}} \ln \frac{r}{R_{\text {out }}}$ (2) And $\mathrm{V}=\mathrm{V}_{+}-\mathrm{V}=\mathrm{V}_{\text {(inn) }}-\mathrm{V}_{\text {(out) }}=\mathrm{Q} / 2 \pi \varepsilon_{\text {oL }} \ln \mathrm{R}_{\text {out }} / \mathrm{R}_{\text {inn }}$ (3) and capacitances for cylindrical geometries are: 
$\bar{C}_{g} \equiv \frac{Q}{V}=\frac{2 \pi \varepsilon_{0} d_{l}}{\ln \frac{R_{\text {out }}}{R_{\text {in }}}} \mathbf{( 4 )}$ which "K" is the dielectric constant of the system ${ }^{65}$.

$\Delta \mathrm{V}_{\text {(inn-out) }}$, is positive quantities because $2 \mathrm{kln}$ $\left(R_{\text {inn }} / R_{\text {out }}\right)$ is a positive quantities these because outer layers are at a higher potential than the inner layers.

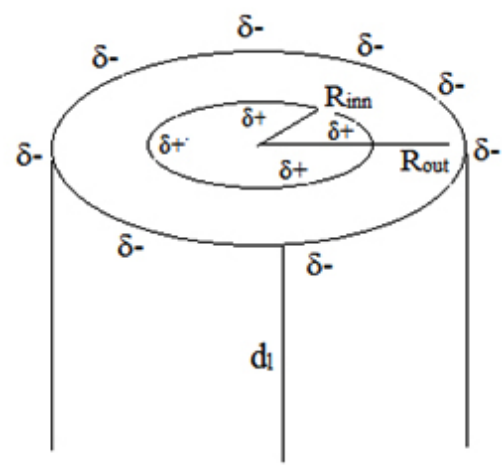

\section{Scheme 1}

In our systems the calculation of the nano-cylindrical capacitors can be obtained from the electrical potential in the spaces between two coaxial cylinders of radii $R_{\text {inn }}$ and $R_{\text {out }}$ and finite length $d_{1}$ in the $z$ direction, $0 \leq \mathrm{z} \leq \mathrm{d}$. We supposed the geometrical capacitances in our systems are a function of $d_{1}, R_{\text {inn }} R_{\text {out }}$ or $C_{g}$ $=F\left(d_{1}, R_{\text {inn }} R_{\text {out }}\right)$. Bilalbegovi ${ }^{66-68}$ with a molecular dynamic simulation and extension series based on Bessel functions ${ }^{69}$ has modified and discussed that capacitance around the shorter coaxial cylinders of radios ${ }^{69}$.So the capacitance in our model can be calculated via $C_{g}=\frac{2 \pi R_{i n n} \varepsilon_{0}}{V} \int_{0}^{d_{l}}\left(\frac{\partial \emptyset}{\partial r}\right)_{r=R_{i n n}} d z^{\prime}(5)$ in the Finite Nano-meter scales cylindrical-capacitor based on the classical electrodynamic ${ }^{85}$. For calculation the capacitance for the eq. 4 and eq. 5 for $\left(\bar{C}_{g}, C_{g}\right)$ the potential difference applied between two cylindrical plates $\mathrm{V}=\mathrm{V}_{\text {(inn) }}-\mathrm{V}_{\text {(out) }}$ has been calculated by pop $=$ chelp $\mathrm{G}$ commands ${ }^{76}$.

\section{Computational details}

Calculations were accomplished using GAMESS-US packages ${ }^{72}$. In this work, it has been mainly focused for optimization of each tube with DFT methods ${ }^{73-77}$ consist of the $\mathrm{m06}$ and $\mathrm{m06}-\mathrm{L}^{74}$. The $\mathrm{m} 062 \mathrm{x}^{74}, \mathrm{~m} 06-\mathrm{L}^{74}$, and $\mathrm{m06}-\mathrm{HF}^{74}$ are a unique Meta hybrid $^{74}$ DFT functional with a good correspondence in non-bonded ${ }^{78}$ calculations and are useful for calculating the energies of the distances between two coaxial cylinders of radii $R_{i n n} R_{\text {out }}$ and in the cylindrical capacitor $^{73}$. The Perdew, Burke and Ernzerh of $(\mathrm{PBE})^{75}$ exchange correlation ${ }^{75}$ (XC) functional ${ }^{74,75}$ of the generalized-gradient-approximations ${ }^{95}$ (GGA) are adopted. The lattice constant has been optimized for the atomic coordinate and has done through the minimization of the total energies. For geometries optimizations, all the internal coordinates were relaxed until the Hellmann-Feynman-forces ${ }^{74}$ was less than 0.005 angstrom.

At each inter tube configurations, a single point calculation is carried out and the total energies are recorded. The resulting sliding rotation energy surface isused for fixing our model in a better position.

We employed DFT theories with the van der Waals DFT for modeling the exchange-correlation energies of SBNNTs and SWCNs ${ }^{76}$. The $\{\xi$-basis set $\}$ with polarization ${ }^{76}$ orbital was used for single wall tube ${ }^{76}$.

For non-covalent approaches, DFT methods disable for describing van der Waals ${ }^{73,77}$. The other functional are correctly insufficient for showing the correlation and exchange energies in non-bonded medium-ranges distances. Furthermore, recent study has illustrated that the medium-range exchange ${ }^{78,79}$

Table1: Gap energy, Fermi level and interaction energy between two layers of DWBNNTs

\begin{tabular}{lclccc}
\hline $\begin{array}{l}\text { SWBNNTs } \\
@ S W B N N T s\end{array}$ & $\begin{array}{c}\text { LUMO/HOMO } \\
\text { Gap(kJ/mol) }\end{array}$ & $\begin{array}{l}\text { Fermi energy } \\
\text { level (a.u.) }\end{array}$ & $\mathbf{I}_{\mathrm{n}} \mathbf{R}_{\text {out }} / \mathbf{R}_{\text {inn }}|\Sigma \mathbf{Q}| \begin{array}{c}\Delta \mathbf{E}_{\mathrm{s}}=\mathbf{E}_{\text {total }}- \\
\left(\mathbf{E}_{\text {inn }}+\mathbf{E}_{\text {out }}\right)\end{array}$ \\
\hline$(5,5) @(7,7) 216$ & 7.96 & -0.393580 & 0.85 & 0.53 & $-0.44 \mathrm{eV}$ \\
$(5,5) @(8,8)$ & 9.67 & -0.393452 & 0.95 & 0.89 & $-0.53 \mathrm{eV}$ \\
$(5,5) @(9,9)$ & 7.55 & -0.393364 & 1.02 & 0.71 & $-0.31 \mathrm{eV}$ \\
$(5,5) @(10,10)$ & 8.75 & -0.393375 & 1.08 & 0.15 & $-0.22 \mathrm{eV}$ \\
\hline
\end{tabular}


energies leads to the large systematic errors ${ }^{78}$ in the prediction of molecular propertie ${ }^{79}$.

We further calculated the interaction energies between two coaxial cylinders of radii " $R_{\text {inn }}$ " and " $R_{\text {out }}$ " for SBNNTs and SWCNs in the structures. The dielectric permittivities as function of dielectric size were determined via
Abinito calculation ${ }^{78-79}$. The interaction energies for capacitor were calculated via an extended huckel method in all items according to the eq.6. $\Delta \mathrm{E}_{\mathrm{s}}(\mathrm{eV})=\left\{\mathrm{E}_{\text {total }}-\left(2 \mathrm{E}_{\text {SWNT }}+\mathrm{E}_{\text {SWBNNT }}\right)\right\}+\mathrm{E}_{\mathrm{BSSE}}$ (6) Where the " $\Delta \mathrm{E}$ " is the stability energy of capacitor.

The charge transfer and electrostatic potential-derived also calculated using the "Merz-
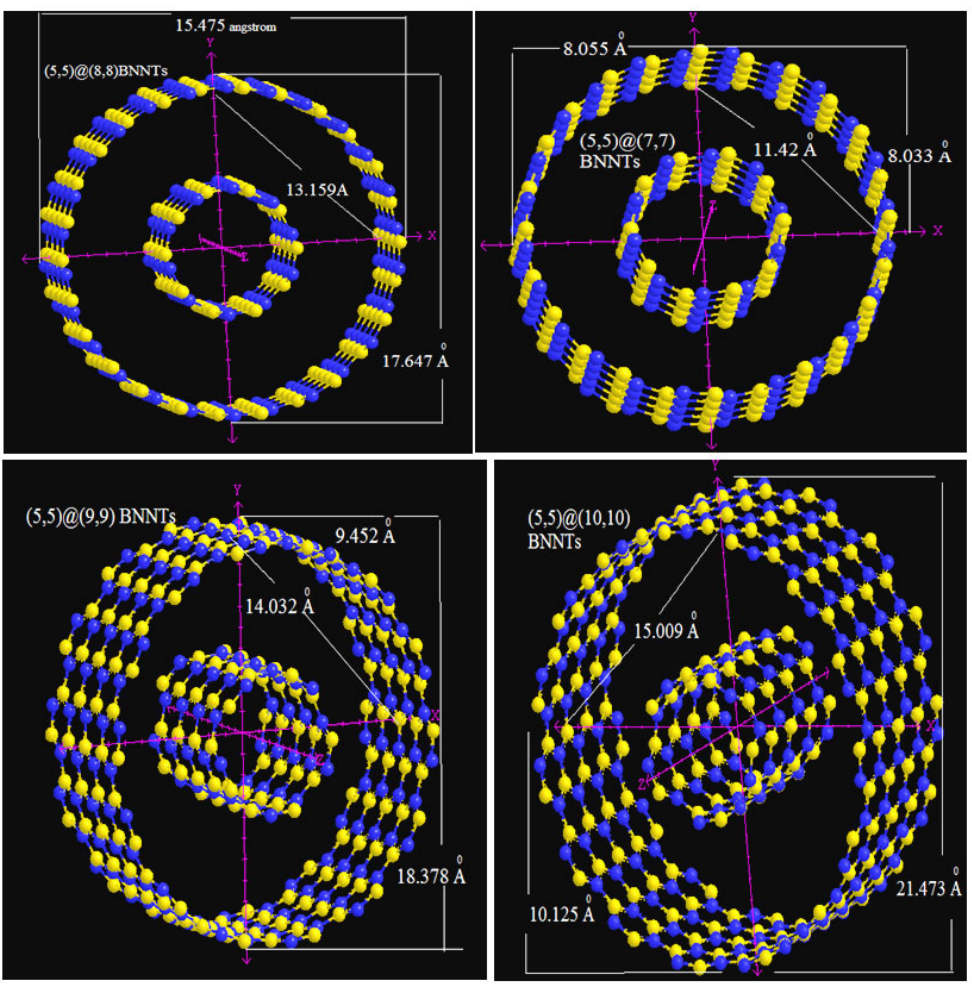

Fig. 1: Dimension of MWBNNTs with various diameters and chiralities

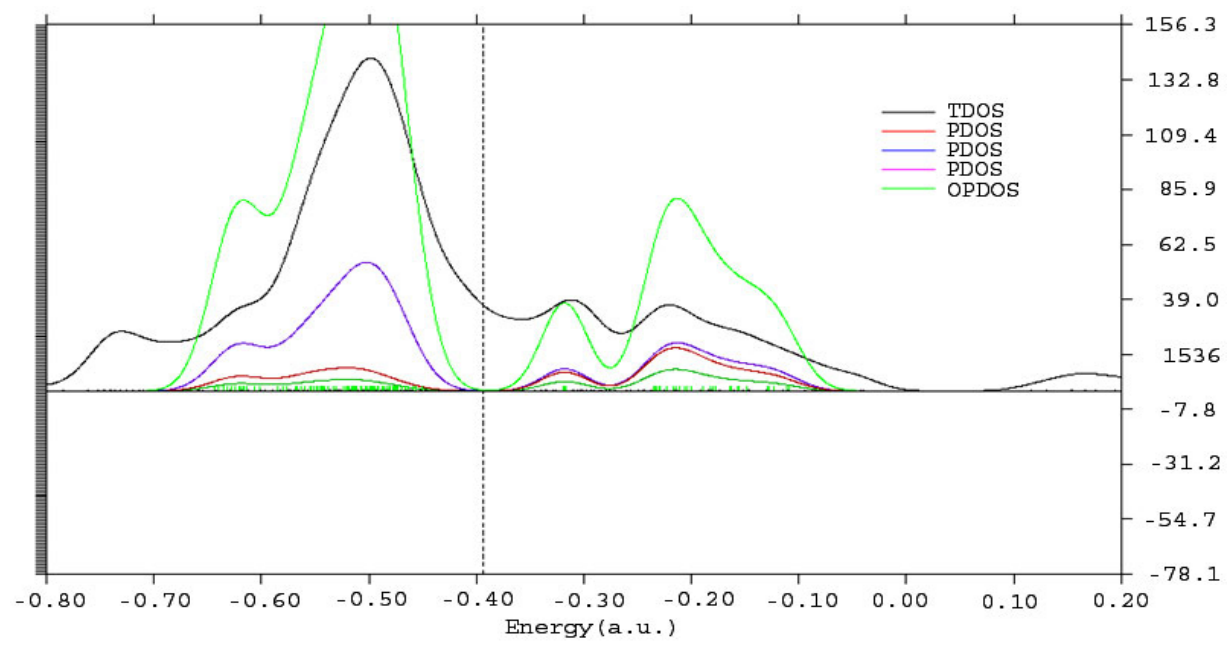

Fig. 2: TDOS, PDOS, OPDOS versus energy for $(5,5) @(8,8)$ BNNTs 
Kollman-Singh ${ }^{80}$, Chelp $^{81}$, ChelpG $^{82}$ and MESP ${ }^{83-84}$.

The MESPs are calculated and distributed at large number of grid points ${ }^{84}$ in a cube regularly.

The representative ${ }^{85}$ atomic charges ${ }^{86}$ would be computed as averages values over a few molecular conformations for the molecules ${ }^{86,87}$.

The electron densities Both of \&Laplacian ${ }^{88}$ and Gradient norms ${ }^{89}$, value of orbital wavefunction ${ }^{90}$, electron spin densities ${ }^{88}$, localization function $(E L F)^{89}$, localized orbital locator ${ }^{90}$,electrostatic potential $^{88}$ from nuclear- atomic-charges, electron total electrostatic potential (ESP) ${ }^{90}$, and the exchange-correlation density, correlation hole and correlation factor, Average local ionization energy using the Multifunctional-Wave-function Analyzer ${ }^{88-90}$.

\section{RESULT AND DISSCUSSION}

We first consider the h-BN sheet and 3D $\mathrm{BN}$ tubes in various diameters and chirality where the $(5,5) @(8,8)$ structure is found as a stable form compared to other forms. The stability depends on the distance between inner radius " $\mathrm{R}_{\mathrm{inn}}$ ", and outer radius " $\mathrm{R}_{\text {out }}$ in one hand and the chirality on other hand Table1. The minimum energies are calculated based on eq. 9 in terms of the total energy of the optimized structures and are listed in Table1.

The differences in the band structure and Fermi ${ }^{88}$ level energy of different tubes have been calculated. Furthermore, we have presented the

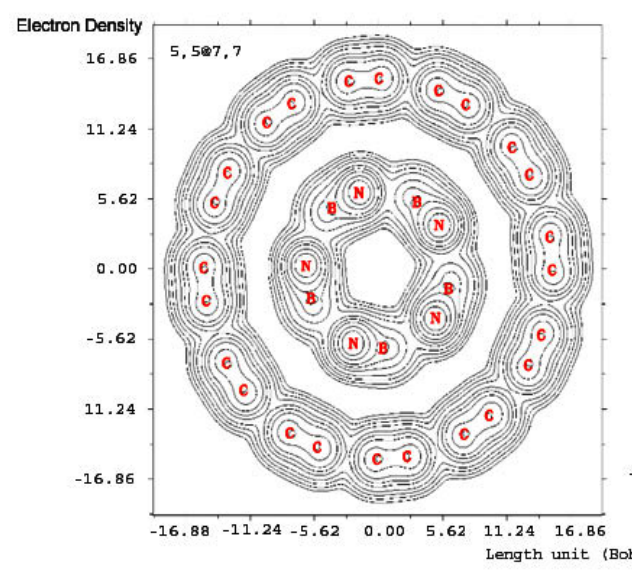

number of states in unit energy interval through density of states (DOS) (Fig.1). DOS ${ }^{88}$ was plotted as a curved map and we have considered those graphs as a tool for analyzing the nature of electronic structure in our systems ${ }^{88,89}$. The original total DOS (TDOS $^{89}$ of our system was calculated based on References $\{108-110\}^{108-110}$ in Figs. 2, 3, 4and 5.

The interaction energies for capacitors were calculated in all items according to the eq. $6 \Delta \mathrm{E}_{\mathrm{s}}(\mathrm{eV})$ $=\left\{\mathrm{E}_{\mathrm{c}}-\left(2 \mathrm{E}_{\text {Dopant }-\mathrm{G}}+\mathrm{E}_{\mathrm{n}-{ }_{\mathrm{BN}}}\right)\right\}+\mathrm{E}_{\mathrm{BSSE}}$ Where the " $\Delta \mathrm{E}_{\mathrm{s}}$ " is the stability energy of capacitor.

The wall buckling has been defined as the differences between the mean radiuses ${ }^{91}$ of the cylinders consisting of the $\mathrm{B}$ and $\mathrm{N}$ atoms. It has been shown that the buckling rapidly increases ${ }^{92}$ with decreasing of the tube diameter ${ }^{91}$ and are independent of the chirality ${ }^{92,93}$.

The $(5,5)$ SWBNNTs in the form of inner cylindrical are semiconductors due to the existence of an energy gap in the range of $(7.55-9.67) \mathrm{KJ} / \mathrm{mol}$ (Table1) which are between the valence band and the conductor $^{93}$ band. Those armchair tubes have a direct band gap, similar to the BN structure ${ }^{94}$.

The projected local density of states (PDOS) of $\mathrm{B}$ and $\mathrm{N}$ are plotted in Fig.2 together with the total DOS for comparison. The data for interaction energy shows that the $(5,5) @(8,8)$ DWBNNTs have a stable form comparing to other systems which yields a suitable charge transfer for the Nano capacitor $^{92,93}$.

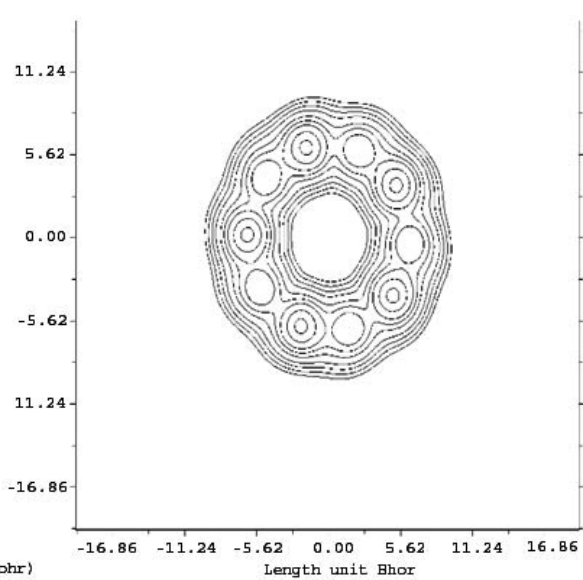

Fig. 3: Counter maps of density for $(5,5) @(7,7)$ BNNTs 
The calculated values of charge transfer for $(5,5) B-N-N T s @(7,7) B-N-N T s,(5,5) B-N-N T s @$ $(8,8) B-N-N T s$ and $(5,5) B-N-N T s @(9,9) B-N-N T s$ and $(5,5) B-N-N T s @(10,10) B-N-N T s$ from inner to outer tubes is found to be $0.53,0.89$, and 0.71 and
0.15 electrons respectively, which is an acceptable value (Table1) and it is small for the $(5,5) @(10$, 10) structures due to their unstable forms (Fig 3 ).

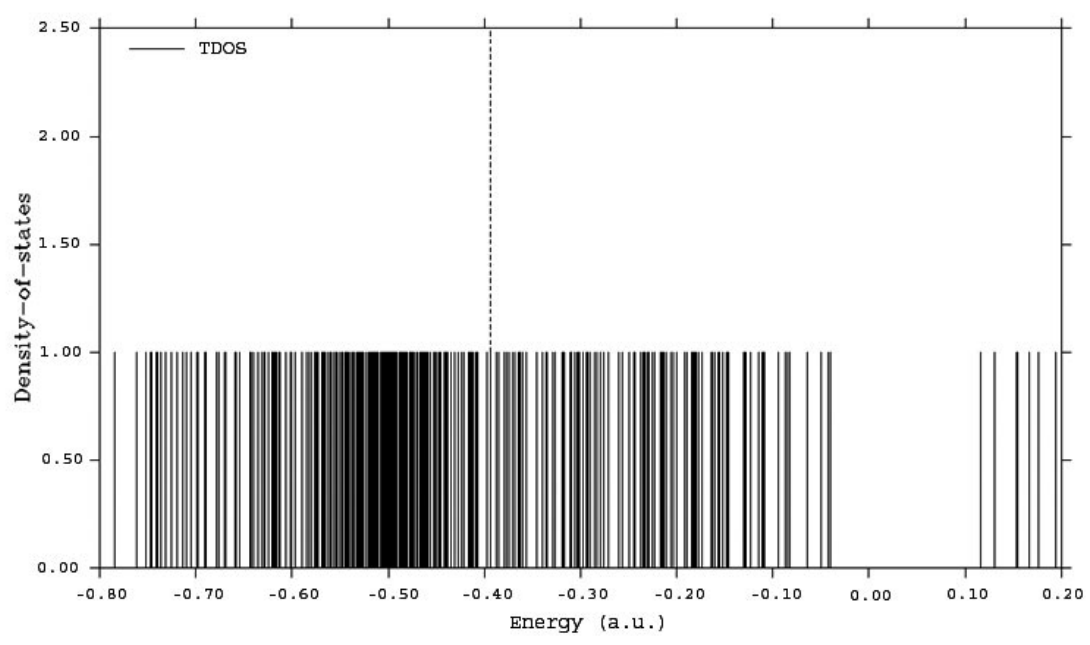

Fig. 4: Density of State versus energy for $(5,5) @(8,8)$ BNNTs

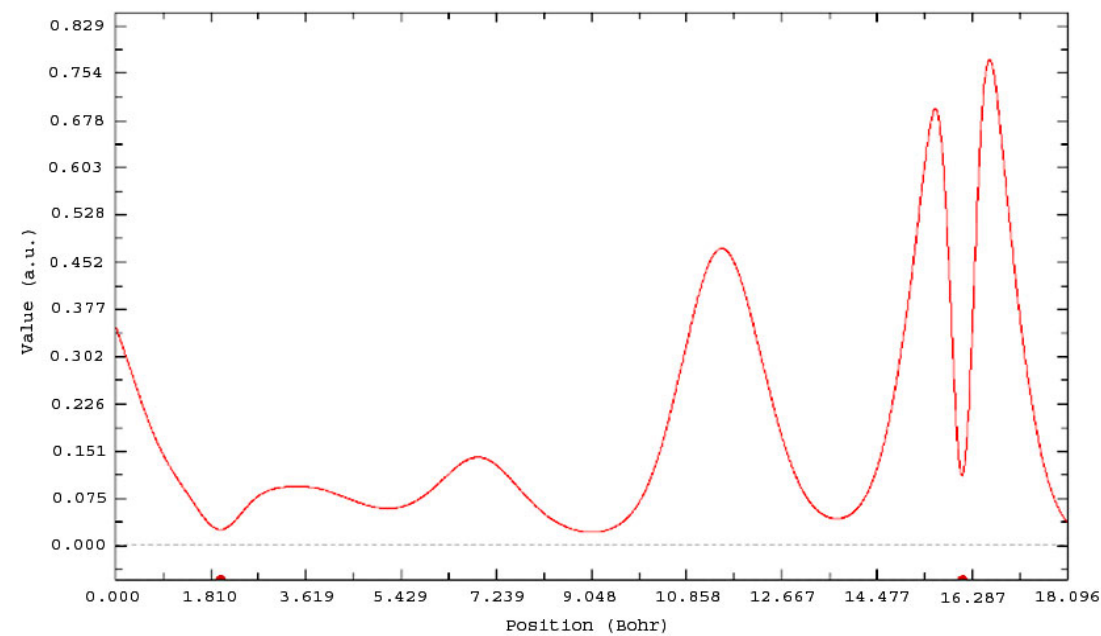

Fig. 5: Energy versus position (Bohr)

Table2. The dielectric constant and $\mathrm{C}_{\mathrm{g}}$ for various DWBNNTs

\begin{tabular}{|c|c|c|c|c|c|c|}
\hline $\begin{array}{l}\text { SWBNNTs @ } \\
\text { sWBNNTs }\end{array}$ & $\mathrm{I}_{\mathrm{n}} \mathbf{R}_{\text {out }} / \mathbf{R}_{\mathrm{inn}}$ & $|\Sigma Q|$ & $\sum_{\substack{\text { out }-i n n \\
-V_{1}}} V_{2}$ & $C_{q} \times 10^{20}$ & $\overline{\frac{2 \pi \varepsilon_{0} d_{l}}{\ln \frac{R_{\text {out }}}{R_{\text {inn }}}}=\bar{C}_{g} \times 10^{20}}$ & $k=\frac{\bar{C}_{g}}{C_{q}}$ \\
\hline$(5,5) @(7,7)$ & 0.85 & 0.53 & 1.23 & 6.8 & 9.8 & 1.44 \\
\hline$(5,5) @(8,8)$ & 0.95 & 0.89 & 1.65 & 8.63 & 8.77 & 1,016 \\
\hline$(5,5) @(9,9)$ & 1.02 & 0.71 & 1.43 & 7.94 & 8.17 & 1.029 \\
\hline$(5,5) @(10,10)$ & 1.08 & 0.15 & 0.78 & 3.07 & - & - \\
\hline
\end{tabular}


It is indicated in this study that the DWBNNTs can be used as a capacitor due to the semiconductor character, metallic behavior and dielectric function of SWBNNTs. Surprisingly, the optical properties ${ }^{93}$ of the SWBNNTs optimized by Guo ${ }^{94}$ found that the dielectric function ${ }^{93}$ could also be divided into two spectral regions ${ }^{94}$, namely, the high and the low energy region -energies these systems ${ }^{94}$.

We show that for the $(5,5) @(8,8)$ the distribution of the accumulated charges in the inner tubes are quantum-mechanically spilled outwards clearly. The capacitance $\left(\mathrm{C}_{\mathrm{q}}\right.$ and $\mathrm{C}_{\mathrm{g}}$ ) and dielectric constant of the various capacitors have been calculated based eq. 4 and are listed in table. 2 .

\section{CONCLUSION}

This work has been investigated the electric polarization and capacitance in a nano-scale coaxial-cylindrical-capacitors which is made of DW boron nitride, $(n, n) @(m, m)$, using ONIOM model including density-functional and semi empirical methods with the enforced Fermi-energies difference scheme. Despite the fact that the SWBNNTs@ SWBNNTs are a cylindrical capacitor, it has a very sensitive electrical storage compared to SWCNTs @ SWCNTS.

\section{REFERENCES}

1. M.Topsakal, S. Ciraci, Phys. Rev. B, 2012 , 85, 045121. 18-20

2. Klein, D. L.; Roth, R.; Lim, A. K. L.; Alivisatos, A. P.;McEuen, P. L. Nature (London) 1997,389, 699

3. Ryo Nakanishi, , Ryo Kitaura, , Jamie $\mathrm{H}$. Warner, Yuta Yamamoto ,, Shigeo Arai, Yasumitsu Miyata, \& Hisanori Shinohara , SCIENTIFIC REPORTS I 3 : 1385 2013, DOI 10.1038 /srep01385

4. Dai, H. J.; Rinzler, A. G.; Nikolaev, P.; Thess, A.; Colbert, D. T.;Smalley, R. E. Chem. Phys. Lett. , 1996, 260, 471

5. Sinnott, S. B.; Andrews, R.; Qian, D.; Rao, A. M.; Mao, Z.; Dickey,E. C.; Derbyshire, F. Chem. Phys. Lett. 1999, 315, 25,

6. Cheung, C. Li.;Kurtz,A.;Park,H.;Lieber,C.M.; J. Phys. Chem. B, 2002, 106, 24292433

7. Z. M. Li, Z. K. Tang, H. J. Liu, N. Wang, C. T. Chan, R. Saito, S. Okada, G. D. Li J. S. Chen, N. Nagasawa, and S. Tsuda, Phys. Rev. Lett. 2001, 87, 127401

8. Z. K. Tang, L. Zhang, N. Wang, X. X. Zhang, G. H. Wen, G. D. Li, J. N. Wang, C. T. Chan, and P. Sheng, Science, 2001,292, 2462,

9. D. Connétable, G. M. Rignanese, J. C. Charlier, and X. Blase, Phys. Rev. Lett. 2005, 94, 015503

10. P. M. Ajayan and S.lijima, Nature, 1992, 358, 23

11. L. F. Sun, S. S. Xie, W. Liu, W. Y. Zhou, and Z.
Q. Liu, Nature, 2000,403, 384

12. N. Wang, Z. K. Tang, G. D. Li, and J. S. Chen, Nature 408, 50 2000. L. C. Qin, X. Zhao, K. Hirahara, Y. Miyamoto, Y. Ando, and S. lijima, Nature 2000,408, 50

13. X. Zhao, Y. Liu, S. Inoue, T. R. Suzuki, O. Jones, and Y. Ando, Phys. Rev. Lett. 2004,92, 125502

14. T. Hayashi, Y. A. Kim, T. Matoba, M. Esaka, K. Nishimura, T. Tsukada, M. Endo, and M. S. Dresselhaus, Nano Lett. 2003 3, 887

15. A. Rubio, J. L. Corkill, and M. L. Cohen, Phys. Rev. 1994, B 49, 5801

16. N. G. Chopra, R. J. Luyken, and K. Cherrey, Science, 1995, 269, 966

17. M.Monajjemi J Mol Model. 20(11):2507. doi: 10.1007/s00894-014-2507-y. Epub Oct 31, 2014

18. S. Okada, S. Saito, and A. Oshiyama, Phys. Rev. 2002, B 65, 165410

19. Xiang, H., Yang, J., Hou, J. \& Zhu, Q. Phys. Rev. 2003, B 68, 035427

20. Chen, Y., Zou, J., Campbell, S. J. \& Le Caer, G. Appl. Phys. Lett. 2004,84, 2430-2432,

21. Kitaura, R. et al. Angew. Chem. Int. Ed. 2009, 48, 8298-8302

22. Zhao, X.,Ando, Y., Liu, Y., Jinno,M.\&Suzuki, T. Phys. Rev. Lett. 2003, 90, 187401

23. Shiozawa, H. Adv. Mater. 2008, 20, 14431449

24. Ando, Y. Chem. Phys. Lett. 2000,323, 580- 
585

25. U. D. VenkateswaranPhys. Rev. B, 2002, 65, 054102

26. GugangChen,S. Bandow,E. R. Ma rgine,C. Nisoli,A. N. Kolmogorov, Vincent H. Crespi,R. Gupta,G.U. Sumanasekera,S. lijima, and P. C. Eklund, PHYSICAL REVIEW LETTERS, 2003,90, $25,257403,1-4$

27. A. Loiseau , F. Willaime , N. Demoncy, G. Hug and H. Pascard , Phys. Rev.Lett. 1996, 76, 4737

28. M. Radosavljevic, J. Appenzeller, V. Derycke , R. Martel, Ph. Avouris, A. Loiseau, J.-L. Cochon and D. Pigache, Appl. Phys. Lett. 2003,82,4131

29. Y. Miyamoto, A. Rubio, M. L. Cohen and S. G. Louie, Phys. Rev. B,1994, 50,4976

30. Y. H. Kim, K. J. Chang and S. G. Louie, Phys. Rev. B, 2001,63, 205408

31. K. H. Khoo, M. S. C. Mazzoni and S. G. Louie, Phys. Rev. B 2004,69, 201401R

32. C. W. Chen, M. H. Lee and S. J. Clark, Nanotechnology, 2004, 15, 1837

33. M. Ishigami, J. D. Sau , S. Aloni, M. L. Cohen and A. Zettl, Phys. Rev. Lett. 2005, 94,56804

34. Y. Miyamoto, A. Rubio, M.L. Cohen and S. G. Louie, Phys. Rev. B, 1994,50, 4976

35. E. J. Mele and P. Kral, Phys. Rev. Lett. 2002,88,56803

36. S. M. Nakhmanson, A. Calzolari,V. Meunier, J. Bernholc and M. B. NardelliPhys. Rev. B, 2003,67, 235406

37. X. Bai, D. Golberg, Y. Bando, C. Zhi, C. Tang, M. Mitome and K. Kurashima, Nano Lett.1997, 7, 632

38. Kazuyuki Uchida, Susumu Okada, Kenji Shiraishi and Atsushi OshiyamaJ. Phys.: Condens. Matter, 2007, 19365218

39. Phys. Rev. B 76, 155436 - Published 30 October Kazuyuki Uchida, Susumu Okada, Kenji Shiraishi, and Atsushi Oshiyama, 2007

40. Monajjemi, M.; Boggs, J.E. J. Phys. Chem. A, 2013,117,1670 "1684

41. Mollaamin, F.; Monajjemi, M, Journal of Computational and Theoretical Nanoscience. 2012, 9 (4) 597-601

42. Mollaamin, F.; Varmaghani, Z.; Physics and Chemistry of Liquids. 2011,49318
43. Monajjemi, M. Chemical Physics. 2013,425, 29-45,

44. Monajjemi, M.; Wayne Jr, Robert. Boggs, J.E. Chemical Physics. 2014,433, 1-11

45. Monajjemi, M.; Mollaamin, F. Journal of Computational and Theoretical Nanoscience, 2012, 9 (12) 2208-2214

46. Monajjemi, M. Falahati, M.; Mollaamin, F.; Ionics, 2013, 19, 155-164

47. Monajjemi, M.; Mollaamin, F. Journal of Cluster Science, 2012, 23(2), 259-272

48. Tahan, A.; Monajjemi, M. Acta Biotheor, 2011,59, 291-312, (2011)

49. Honaparvar, B.; KhaliliHadad ,B.; Ilkhani ,AR.; Mollaamin, F.African Journal of Pharmacy and Pharmacology .2010, 4(8), 521-529

50. Mollaamin, F.; Monajjemi, M.Physics and Chemistry of Liquids , 2012,50, 5, 2012, 596-604

51. Monajjemi, M.; Khosravi, M.; Honarparvar, B.; Mollaamin, F.; International Journal of Quantum Chemistry, 2011, 111, 27712777

52. Monajjemi, M. TheorChemAcc, 2015,134:77 DOI 10.1007/s00214-015-1668-9

53. Jalilian, H.; Monajjemi, M. Japanese Journal of Applied Physics. 2015,54, 085101

54. Monajjemi, M.; Khaleghian, M.; Mollaamin, F. Molecular Simulation. 2010, 36, 11, 865-870

55. Soofi, N.S., Monajjemi, M, Oriental Journal of Chemistry. 2016, 32, 11, 2327-2345

56. Elsagh, A., Zare, K., Monajjemi, M. Oriental Journal of Chemistry, 2016, 32, 5, 25852598

57. Raoufi, F.;Aghaee, H.;Monajjemi, M. Oriental Journal of Chemistry,2016,32, 4, 18391858

58. Rahimi, A.;,Monajjemi, M, Oriental Journal of Chemistry, 2016, 32, 6, 2957-296559. Monajjemi, M.Struct Chem. 2012, 23,551580

60. Monajjemi, M.; Rajaeian, E.; Mollaamin, F.; Naderi, F.; Saki, S. Physics and Chemistry of Liquids. 2008, 46 (3), 299-306

61. Mollaamin, F.; Gharibe, S.; Monajjemi, M. Int. J. Phy. Sci, 2011, 6, 1496-1500

62. S. lijima and T. Ichihashi, Nature,1993,363, 603

63. D.S. Bethune, e.B. Kiang, M.S. de Vries, G. 
Gorman, R Savoy, J. Vazquez, RBeyers, Nature 1993, 363, 605

64. J.W. Mintmire, B.I. Dunlap, and C.T. White, Phys. Rev. Lett. 1992, 68, 631

65. R Saito, M. Fujita, G. Dresselhaus, and M.S. Dresselhaus, Appl. Phys. Lett. 1992, 60, 2204

66. G. Bilalbegovic, Phys. Rev. B,1998,58, 15412

67. G. Bilalbegovic, Solid State Commun. (2000)

68. G. Bilalbegovic, Computational Materials Science, 2000, 18, 333-338

69. I. S. Gradshteyn and I. M. Ryzhik, Table of Integrals, Series, and Products (Academic Press, New York, (1965)

70. W. A. de Heer, Rev. Mod. Phys., 1993,65, 611

71. S. Wang and M. Grifoni, Phys.Rev.B, 2008,77, 085431

72. M.W.Schmidt, K.K.Baldridge, J.A.Boatz, S.T.Elbert, M.S.Gordon, J.H.Jensen, S.Koseki, N.Matsunaga, K.A.Nguyen 2004,14(11) 1347-1363

73. Yan Zhao, Donald G. Truhlar, TheorChem Account, 2008, 120,215-241

74. W. Kohn, L. J. Sham, Phys. Rev. 140 A ,1965,1133-1138

75. J.P. Perdew, K.Burke, Ernzerhof, Phys. Rev. Lett. 1996,77, 3865-3868

76. D. L. Klein, R. Roth, A. K. L. Lim, A. P. Alivisatos, and P. L. McEuen, Nature (London) 1997,389 699

77. Yan Zhao, Donald G. Truhlar, Accounts of
Chemical Research, 2008,41(2) 157-167

78. C.E.Check, T.M.Gilbert, J. Org. Chem, 2005,70,9828-9834

79. S.Grimme, Seemingly. Angew. Chem., Int. Ed. 2006,45,4460-4464

80. B.H. Besler, K.M. Merz, P.A. Kollman, J. comp. Chem. 1990, 11,431

81. L.E. Chirlian, M.M. Francl, J.comp.chem. $1987,8,894$

82. Brneman GM, WibergKB ,J. Comp Chem, 1990, 11: 361

83. Martin F, ZipseH .J Comp Chem. 2005,26: 97 ,105

84. Monajjemi M, Lee VS, Khaleghian M, Honarparvar B, Mollaamin F ,J. Phys. Chem. C, 2010,11415315

85. M. MonajjemiM ,KhaleghianM J ClustSci, 2011,22:673-692

86. Monajjemi M Struct. Chem. 2012, 23: 551

87. Balderchi, A, Baroni S, Resta RPhys. Rev. Lett 1998,61: 173

88. Tian Lu, Feiwu Chen, ActaChim. Sinica, 2011,69, 2393-2406

89. Tian Lu, Feiwu Chen, J. Mol. Graph. Model. 2012, 38, 314-323

90. Tian Lu, Feiwu Chen, Multiwfn: J. Comp. Chem. 2012, 33, 580-592

91. X. Blase, Phys. Rev. Lett. 1994,72, 1878

92. E. Hernández, C. Goze, P. Bernier, and A. Rubio, Phys. Rev. Lett. 1998,80, 4502

93. L. Wirtz, A. Rubio, R. A. Concha, and A. Loiseau, Phys. Rev. B, 2003, 68, 045425

94. G.Y. Guo, J.C. Lin, Phys. Rev. B, 2005, 71, 165402 